\title{
A comparison of five questionnaires to assess alcohol consumption in a Mediterranean population
}

\author{
L Serra-Majem 1,2,* , JF Santana-Armas ${ }^{1}$, L Ribas ${ }^{2}$, E Salmona ${ }^{1}$, JM Ramon ${ }^{2}$, J Colom ${ }^{3}$ and \\ L Salleras ${ }^{2,3}$ \\ 'Department of Clinical Sciences, University of Las Palmas de Gran Canaria, PO Box 550, Las Palmas 35080, \\ Canary Islands, Spain: ${ }^{2}$ Community Nutrition Research Center, Barcelona Science Park, University of Barcelona, \\ Spain: ${ }^{3}$ Department of Health and Social Security, Autonomous Government of Catalonia, Spain
}

Submitted 2 July 2001: Accepted 4 December 2001

\begin{abstract}
Objective: To evaluate the reliability of alcohol consumption measured by five different dietary methods in the Catalan Nutrition Survey (1992-1993).

Design: The different questionnaires used were: (1) two 7-day food records, (2) five items addressing alcoholic beverages in a 76-item food-frequency questionnaire, (3) a 1-week recall of 13 alcoholic beverages, (4) a 13-item alcoholic beverages frequency questionnaire and (5) two 24-hour recalls.

Subjects: Ninety-three of the 120 adults (aged 20 to 70 years) initially recruited completed the questionnaires and provided a peripheral blood sample.

Results: Mean daily alcohol intakes as assessed by the questionnaires were very similar. Beer was the alcoholic beverage most frequently consumed, followed by wine and liquor. Eighty-two per cent of the study population consumed less than $20 \mathrm{~g}$ of alcohol per day. No biochemical parameters were significantly correlated with alcohol intake estimated from the questionnaires.

Conclusions: We found a satisfactory level of reproducibility and validity in the pattern of alcohol consumption across different levels and types of alcoholic beverage intake. We also found that the self-administered 13-item questionnaire and the 1-week recall were the best techniques to measure moderate or low alcohol consumption, suggesting that the two methods are the most suitable to assess overall alcohol intake in the general population.
\end{abstract}

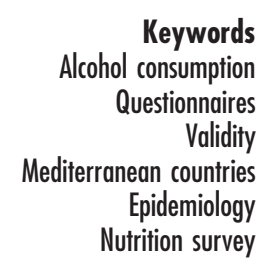

Alcohol is an age-old drug, the most widely used psychoactive substance in our society and an important determinant of several common diseases in humans. It occupies a very important place in our culture and in the economy. Its consumption has been traditionally high in Mediterranean countries, particularly those in the western part of the Mediterranean Basin ${ }^{1}$. The pattern and problem of drinking alcohol are distinctive in these countries (regular daily intake, especially wine, is more common than binge ingestion) and evaluation of alcohol intake has focused too much on the alcohol-dependent population and the assessment of excessive alcohol intake. Low and moderate alcohol consumption may increase or reduce the risk of cancer, heart and liver diseases ${ }^{2}$, highlighting the need for accurate and practical methods to assess low levels of alcohol intake.

It has been suggested that moderate alcohol consumption protects against coronary heart disease, and that this may at least partly explain the low cardiovascular disease incidence and mortality in Mediterranean countries. However, the interpretation of this observed relationship is still controversial ${ }^{3,4}$. A number of epidemiological and biologically plausible mechanisms of action have been formulated, including the relationship between alcohol drinking and high-density lipoprotein cholesterol apolipoproteins, fibrinolysis, coagulation factors and blood pressure, but none has yet been firmly established. Most authors consider low or moderate alcohol consumption to be a protective factor for certain diseases and syndromes ${ }^{5}$. However, on a public health scale, drinking alcohol is significantly linked to the risk of liver cirrhosis and a few cancer sites, including those of the upper digestive and respiratory tract, whose incidence and mortality are exceedingly high in western Mediterranean countries ${ }^{6}$.

Consuming alcohol is a widespread habit in Spain, especially at mealtimes and particularly in males ${ }^{7-9}$. However, alcohol consumption including wine and spirits has been decreasing in Spain during the last decade ${ }^{10}$.

Assessment methods for alcohol consumption at an individual level may include various procedures with different measures of alcohol intake. Estimates of alcohol consumption could be made using self-reported infor- 
mation, and by interview, proxy reports and blood analysis.

The aim of this study was to select a simple method for measuring alcohol consumption in a cohort study that analyses the relationship between levels of alcohol consumption and liver disease incidence in Spain. In this study, we compared and evaluated five questionnaires: (1) two 7-day dietary records (14-day diet record), (2) a fiveitem questionnaire included in a food-frequency questionnaire, (3) a 1-week recall, (4) a 13-item self-reported alcoholic beverages frequency questionnaire, and (5) two 24-hour recalls on non-consecutive days. In this paper, the comparison of the mentioned procedures was not a rigorous test of reliability and validity of the different methods used to assess alcohol consumption, but rather a comparison of the questionnaires most frequently used in studies. Reported alcohol intake and consumption of alcoholic beverages were compared in a random sample of adults from Catalonia, a Mediterranean region of Northeast Spain.

\section{Material and methods}

\section{Study design}

The general design of the Catalan Nutrition Survey has been published elsewhere ${ }^{11}$. A random sample of individuals completed five different interviews and selfadministered food-frequency questionnaires, between 1992 and 1993, with a time sequence of completion as defined in Fig. 1. Most of the data on alcohol consumption used in this study were collected as a part of the survey on nutritional status assessment in the study population.

\section{Subjects}

A total of 120 adults ( 62 men and 58 women, aged 20 to 70 years) were recruited from the initial random sample of the Catalan Nutrition Survey. A sample of peripheral blood was obtained from each participant, for a total of 100 people out of 120 initially recruited.

\section{Questionnaires}

Five different questionnaires were gathered from May 1992 to June 1993, as follows.

1992 1993

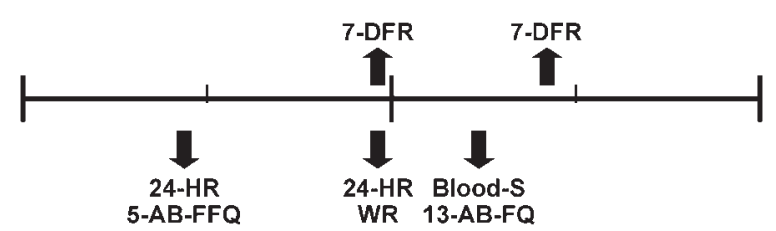

Fig. 1 Time sequence of completion of two 7-day food records (7-DFR), a five-item alcoholic beverages in a food-frequency questionnaire (5-AB-FFQ), a 13-item self-reported alcoholic beverages frequency questionnaire (13-AB-FQ), two 24-hour recalls (24-HR) and a 1-week recall (WR); and blood sample (Blood-S) among 93 adults in Catalonia, Spain
1. Two 7-day food records obtained by trained interviewers were collected from November 1992 to June 1993. This was chosen as the reference method because it is not affected by memory and provides a direct quantitative measure of intake.

2. A 76-item food-frequency questionnaire was used in the first part of the Catalan Nutrition Survey. The questionnaire included five alcoholic beverages (beer, wine, vermouths, liquors and spirits) and was gathered by personal interview in the subject's home.

3. A 1-week recall of 13 alcoholic beverages (medium beer, small beer, beer without alcohol, red wine, white wine, sherry, sweet wine, champagne, cocktails, liquors, spirits with coffee, whisky, sweet liquors) was obtained in the second part of the Catalan Nutrition Survey. Taking into account that part of the health information gathered in this survey would be used as a baseline data set for the CINDI Study ${ }^{12}$ in Catalonia, we also applied the assessment method required for this World Health Organization international programme. It consisted of a 1-week consumption recall of 13 alcoholic beverages. This questionnaire was also obtained by personal interview in the subject's home.

4. A 13-item alcoholic beverage frequency questionnaire was self-administered and reviewed by a dietitian just before the blood sample was taken at the local clinic. As for the 1-week recall, a commonly used unit or portion size was specified for each beverage item. Subjects were asked how often, on average, they had consumed the specific amount of each item over the past year. There was a range of possible responses from 'never' to 'daily'.

5. Two 24-hour recalls, the first one being collected from May to June 1992 and the second one from November to December 1992, which were a specific part of the Catalan Nutrition Survey. Results represent the individual average from both 24-hour recalls.

\section{Analytical techniques}

A 12-hour fasting blood sample was obtained from each individual from January to June 1993. Analyses were performed at the Vall d'Hebron General Hospital in Barcelona, Spain. Serum high-density lipoprotein cholesterol (HDL-C) was measured by spectrophotometry, gamma-glutamyl transferase $(\gamma \mathrm{GT})$ was determined by enzymatic colorimetry with an autoanalyser, erythrocyte medium corpuscular volume (MCV) was measured by volumetry (Coulter ${ }^{\circledR}$ and Counter ${ }^{\circledR}$ ), and ferritin levels were determined by immunofluorescence.

\section{Procedure}

The different questionnaires were sequentially administered by trained interviewers and self-administered by the subjects themselves. Participants and interviewers were 
Table 1 Distribution of alcohol intake by questionnaire type

\begin{tabular}{|c|c|c|c|c|c|c|c|c|c|c|}
\hline \multirow[b]{3}{*}{ Alcohol intake } & \multicolumn{10}{|c|}{ Questionnaire } \\
\hline & \multicolumn{2}{|c|}{$\begin{array}{l}\text { Two 7-day food } \\
\text { records }\end{array}$} & \multicolumn{2}{|c|}{$\begin{array}{c}\text { 5-item } A B \text { in } \\
F F Q\end{array}$} & \multicolumn{2}{|c|}{$\begin{array}{l}\text { 13-item self- } \\
\text { reported } \\
\text { AB FQ }\end{array}$} & \multicolumn{2}{|c|}{$\begin{array}{l}\text { Two 24-hour } \\
\text { recalls }\end{array}$} & \multicolumn{2}{|c|}{ 1-week recall } \\
\hline & $n$ & $\%$ & $n$ & $\%$ & $n$ & $\%$ & $n$ & $\%$ & $n$ & $\%$ \\
\hline $0 \mathrm{~g}$ & 20 & 21.9 & 10 & 11.0 & 21 & 21.9 & 43 & 46.5 & 19 & 20.5 \\
\hline $1-19 \mathrm{~g}$ & 56 & 60.3 & 66 & 71.2 & 57 & 61.6 & 36 & 38.4 & 57 & 61.7 \\
\hline$\geq 20 \mathrm{~g}$ & 17 & 17.8 & 17 & 17.8 & 15 & 16.5 & 14 & 15.1 & 17 & 17.8 \\
\hline
\end{tabular}

$\mathrm{AB}$ - alcoholic beverages; FFQ - food-frequency questionnaire; FQ - frequency questionnaire.

instructed to record the different types of questionnaire used in this analysis. The time sequence of completion of these different questionnaire types is represented schematically in Fig. 1.

Conversion of alcohol beverages into grams of alcohol was made using the Spanish and French food consumption tables ${ }^{11}$. The total alcohol intake for each subject was computed as the sum of the different alcoholic beverages reported. Different types of alcoholic beverage were considered separately in order to verify the ability of the questionnaires to assess detailed intake patterns of a variety of alcoholic beverages. Finally, reported intakes of alcoholic beverages such as wine, beer, spirits and liquors were also computed and compared.

Reported alcohol intake measured by the five different methods was compared with serum HDL-C, $\gamma$ GT, MCV and ferritin levels, which are sensitive parameters to alcohol consumption $5,13,14$.

\section{Statistical analysis}

From the initial random sample of 120 people aged 20 to 70 years, 100 completed diet records and 93 people had all the information from the different questionnaires as well as the blood analysis. The Pearson correlation coefficient was used in order to compare alcohol intakes (expressed as $\log _{\mathrm{e}}$ ) for individual subjects from the diet records and questionnaires. We also classified individuals into consumption categories $(0 \mathrm{~g}, 1-19 \mathrm{~g}$ and $\geq 20 \mathrm{~g}$ alcohol per day). Tertiles of consumption from each method were used to examine the degree of misclassification across categories. Pearson correlation coefficients were also used to compare estimations of alcohol intake from diet records and questionnaires with HDL-C, $\gamma \mathrm{GT}, \mathrm{MCV}$ and ferritin levels.

\section{Results}

Comparison of the five measurement methods of alcohol intake under study (classified in three categories of consumption) is shown in Table 1. Alcohol consumption had an approximate log-normal distribution with the tail skewed towards the higher values (mean of $10.22 \pm 8.21 \mathrm{~g}$ alcohol per day). As assessed by diet records, 18\% of the population consumed more than $20 \mathrm{~g}$ of alcohol per day (Table 1) and 2.7\% more than $60 \mathrm{~g}$.

All questionnaires showed that beer was the alcoholic beverage most frequently consumed, followed by wine and liquor (Table 2). The five-item questionnaire detected the highest average of beer consumption and, in this instrument, wine consumption was among the lowest. The two 24-hour recalls detected the highest percentage abstemious.

The Pearson correlation coefficient was used in order to compare alcohol intakes (expressed as $\log _{\mathrm{e}}$ ) for individual subjects from the diet records and questionnaires. Examination of the correlation matrix in Table 3 reveals that all of the questionnaire techniques are highly correlated (between 0.70 and 0.83 ) and that the

Table 2 Alcohol and beverage intakes estimated by the different questionnaires

\begin{tabular}{|c|c|c|c|c|c|c|c|c|c|c|}
\hline \multirow[b]{3}{*}{ Alcohol and beverages } & \multicolumn{10}{|c|}{ Questionnaire } \\
\hline & \multicolumn{2}{|c|}{$\begin{array}{l}\text { Two 7-day food } \\
\text { records }\end{array}$} & \multicolumn{2}{|c|}{$\begin{array}{c}\text { 5-item } A B \text { in } \\
\text { FFQ }\end{array}$} & \multicolumn{2}{|c|}{$\begin{array}{l}\text { 13-item self- } \\
\text { reported } \\
A B F Q\end{array}$} & \multicolumn{2}{|c|}{$\begin{array}{l}\text { Two 24-hour } \\
\text { recalls }\end{array}$} & \multicolumn{2}{|c|}{ 1-week recall } \\
\hline & Mean & SD & Mean & SD & Mean & SD & Mean & SD & Mean & SD \\
\hline Alcohol (g) & 10.1 & 13.7 & 10.6 & 14.1 & 10.5 & 15.4 & 9.4 & 15.4 & 10.5 & 16.2 \\
\hline Beer (cc) & 61.1 & 124.2 & 90.1 & 186.3 & 79.7 & 171.5 & 70.1 & 191.6 & 83.6 & 227.0 \\
\hline Wine (cc) & 56.5 & 80.0 & 49.8 & 73.2 & 53.9 & 82.0 & 46.7 & 77.1 & 54.8 & 83.9 \\
\hline Liquor (cc) & 7.1 & 18.1 & 5.3 & 11.6 & 6.1 & 18.2 & 5.1 & 17.6 & 5.5 & 12.2 \\
\hline
\end{tabular}

SD - standard deviation; $n=93$.

$\mathrm{AB}$ - alcoholic beverages; FFQ - food-frequency questionnaire; FQ - frequency questionnaire. 
questionnaire most highly correlated with the reference method is the 13-item food-frequency questionnaire. Moreover, we found that no biochemical parameters or indicators were significantly correlated to alcohol intake estimates (Table 3). According to the analysis of the correlation matrix based on calculated Pearson correlations, we found that the self-administered 13-item questionnaire was the best method together with the 1-week recall to assess moderate or low alcohol consumption. Results indicate the superior capacity of the 13-item self-administered frequency questionnaire versus the decreased ability of the 24-hour recall method to assess levels of alcohol intake.

The degree of misclassification in tertiles of consumption for each method, compared with the two 7-day dietary record standard, is presented in Table 4 . The best classifications of high consumers (highest tertiles) were obtained by the 1 -week recall and the 13-item alcoholic beverage frequency questionnaire ( $80 \%$ of agreement).

\section{Discussion}

In our country alcohol consumption constitutes a widespread custom. The consumption of alcoholic beverages is one of the main features of the Spanish lifestyle. In 1995, Spain ranked seventh world-wide in the consumption of alcohol, with 10 litres of pure alcohol per capita. This indicates the need to consider harmful health habits within the context of characteristic cultural and social lifestyles that interact with other factors instead of merely focusing on individual behaviour.

Although wine is the traditional Mediterranean alcoholic beverage, beer is the alcoholic beverage most frequently consumed among respondents in our study. This reflects the increasing trend of beer drinking and the decline of wine consumption in Spain over the past two decades. We found no major differences in reporting by beverage type, suggesting that any observed differences of effects according to beverage type are not solely due to different measurement errors.

This study attempts to evaluate the reliability and concordance of five short measures of alcohol and dietary consumption with biochemical parameters. This linkage is an important issue, since most methodological studies of alcohol consumption measures do not use biochemical parameters to estimate the validity of the instruments.

The problem of measuring alcohol consumption, and in particular the question of validity and reliability of selfreports and questionnaires, is a regularly recurring topic in scientific discussions ${ }^{15,16}$.

In past decades several types of retrospective method have been used in alcohol consumption surveys. They can be roughly divided into two categories, the so-called summary measures and the instruments based on recent drinking occasions. The summary measures require the respondent to evaluate aspects of individual behaviour 
Table 4 Degree of misclassification of consumption tertiles from each method compared with the two 7-day food records

\begin{tabular}{|c|c|c|c|c|}
\hline & \multicolumn{4}{|c|}{ Standard method: two 7-day food records } \\
\hline & \multicolumn{2}{|c|}{$\mathrm{P}_{33}\left(1.26 \mathrm{~g}\right.$ alcohol day $\left.{ }^{-1}\right)$} & \multicolumn{2}{|c|}{$\mathrm{P}_{66}\left(11.96 \mathrm{~g}\right.$ alcohol day $\left.{ }^{-1}\right)$} \\
\hline & High tertile & Low tertile & High tertile & Low tertile \\
\hline $\begin{array}{l}\text { 5-item AB in FFQ } \\
\quad P_{33}=1.06, P_{66}=12.75\end{array}$ & $12.5 \%$ & $66.7 \%$ & $72.0 \%$ & $0.0 \%$ \\
\hline $\begin{array}{c}\text { 13-item self-reported AB FQ } \\
P_{33}=0.87, P_{66}=9.60\end{array}$ & $8.3 \%$ & $70.8 \%$ & $80.0 \%$ & $0.0 \%$ \\
\hline $\begin{array}{l}\text { Two 24-hour recalls } \\
\qquad P_{33}=0.00, P_{66}=8.84\end{array}$ & $8.3 \%$ & $83.3 \%$ & $72.0 \%$ & $8.0 \%$ \\
\hline $\begin{array}{l}\text { 1-week recall } \\
\quad P_{33}=2.68, P_{66}=8.31\end{array}$ & $0.0 \%$ & $62.5 \%$ & $80.0 \%$ & $0.0 \%$ \\
\hline
\end{tabular}

over a certain period of time. They are based on questions about frequency and quantity of drinking and are used mostly in American surveys. The recent drinking occasions approach uses self-reports of current consumption. The respondent is asked to list all the drinking events that have occurred in the recent past. This type of method is often used in European surveys. In this work, special attention was given to reach an intermediate measurement method between the American and European approaches.

It is a very well known fact that self-reports on alcohol consumption cannot be trusted in all cases. Deliberate underreporting is an important factor responsible for the large discrepancy between surveys of reported intake and consumption estimates based on sales. However, questionnaires are in general the best method to assess alcohol consumption in all purposes and circumstances. In selfadministered questionnaires the threat and the discomfort imposed by questions on alcohol drinking is probably smaller than in face-to-face interviews ${ }^{17-19}$.

Among methods to assess alcohol intake, diet records provide a relatively direct quantitative measurement and minimally depend on memory ${ }^{20-22}$. However, diet records are impractical to use in typical epidemiological studies. Therefore, most studies have employed self-reports of 'usual' or 'customary' intake to evaluate alcohol consumption. We found that diet records and questionnaire estimates are highly correlated, as shown by the similar estimated mean intakes. Similar consistency between diet records and questionnaire measures of alcohol intake has been observed in other studies ${ }^{23-26}$. A simple measure of overall intake may not distinguish between the patterns of frequent light drinking and infrequent heavy drinking, but the relative stability of individual intake over time indicates that even a single measurement provides useful information. We found that self-administered and interviewer-administered questionnaires can provide estimates of alcohol consumption in the range of moderate intakes. This compares favourably with measures of other exposures that are frequently used in epidemiological studies.
Since the assessment methods of this study are different, correlated sources of error should be small ${ }^{17,27,28}$. The likeliest source of correlated error may arise from participants who intentionally underreport intake for the five methods. In fact, excessive drinking may be perceived as socially inappropriate. Intentional underreporting seems less likely for moderate and low consumption, which is common in Spain and other Mediterranean countries where it is not generally considered undesirable behaviour $^{9,29}$.

The biochemical indicators used in this analysis were not correlated to alcohol intake, although in other studies a relationship has been observed ${ }^{5,13,14}$. The low consumption levels of alcoholic beverages in our sample and also the reduced sample size ${ }^{30}$ could account for this phenomenon.

The choice of the best method for assessing alcohol intake is a difficult issue since all of the methods studied yield reasonable concordance. Moreover, the possibility of biased results could exist due to the exposure of participants to a variety of assessment tools administered over a one-year period (learning bias).

In summary, despite the aforementioned caveats, the self-administered 13-item questionnaire seems to be the best method to assess moderate or low alcohol consumption, followed by the 1 -week recall. In our study no biochemical indicators were correlated to low and moderate alcohol intake estimates.

\section{Acknowledgements}

This study was supported by a research agreement between the Autonomous Government of Catalonia (General Department of Public Health) and the University of Barcelona.

The authors wish to acknowledge Dr Reina GarcíaClosas, Jordi Espuñas, Gemma Salvador, Imma Palma and Lucía Bultó for their help in the field work, and Dr Pilar Chacón and Dr Simó Schwartz for the blood analyses. 


\section{References}

1 La Vecchia C. Alcohol in the Mediterranean diet: assessing risks and benefits. Eur. J. Cancer Prev. 1995; 4: 3-5.

2 International Agency for Research on Cancer (IARC). Alcohol Drinking. IARC Monograph on the Evaluation of Carcinogenic Risk in Humans, Vol. 44. Lyon: IARC, 1988.

3 Miller GJ, Beckles GLA, Maude GH, Carson DC. Alcohol consumption: protection against coronary heart disease and risk to health. Int. J. Epidemiol. 1990; 19: 923-30.

4 Catarino PA. Is there a safe level of drinking?: a student's view. Alcohol \& Alcoholism 1992; 27: 465-70.

5 Macdonald I, ed. Health Issues Related to Alcohol Consumption, 2nd ed. Oxford: ILSI-Europe/Blackwell Science Ltd, 1999.

6 La Vecchia C, Decarli A, Mezzanote G, Cislaghi C. Mortality from alcohol related disease in Italy. J. Epidemiol. Community Health 1986; 40: 257-61.

7 Ministerio de Sanidad y Consumo. Encuesta Nacional de Salud, 1997. Madrid: Ministerio de Sanidad y Consumo, 1997.

8 Regidor E, Gutiérrez JL, de Mateo S. Asociación entre consumo habitual de alcohol y accidentes. Gac. Sanit. 1992; 6: $245-52$.

9 Centro de Investigaciones Sociológicas. Comportamientos de los Españoles ante el Tabaco, el Alcohol y las Drogas. Estudio 1804. Madrid: Centro de Investigaciones Sociológicas, 1990.

10 Gual A, Colom J. Why has alcohol consumption declined in countries of Southern Europe? Addiction 1997; 92(Suppl. 1): S21-31.

11 Serra-Majem L, Ribas L, Ramon JM. Compliance with dietary guidelines in the Spanish population. Results from the Catalan Nutrition Survey. Br. J. Nutr. 1999; 81(Suppl. 2): S105-12.

12 WHO Collaborative Centre for Policy Development in the Prevention of Noncommunicable Disease. Handbook for Process Evaluation in Noncommunicable Disease Prevention. Ottawa/Copenhagen: Health Canada/WHO Regional Office for Europe, 1999.

13 Whitfield JB, Zhu G, Heath AC, Powell LW, Martin NG. Effects of alcohol consumption on indices of iron stores and of iron stores on alcohol intake markers. Alcohol Clin. Exp. Res. 2001; 25: 1037-45.

14 Lakshman R, Tsutsumi M, Ghosh P, Takase S, Anni H, Nikolaeva $\mathrm{O}$, et al. Alcohol biomarkers: clinical significance and biochemical basis. Alcobol Clin. Exp. Res. 2001; 25(Suppl. 5): 67S-70S.
15 Embree BG, Whitehead PC. Validity and reliability of selfreported drinking behavior: dealing with the problem of response bias. J. Stud. Alcohol 1993; 54: 334-44.

16 Redman S, Sanson-Fisher RW, Wilkinson C, Fahey PP, Gibberd RW. Agreement between two measures of alcohol consumption. J. Stud. Alcohol 1987; 48: 104-8.

17 Barbor TF, Stephens RS, Marlatt GA. Verbal report methods in clinical research and alcoholism: response bias and its minimization. J. Stud. Alcohol 1987; 48: 410-24.

18 Block G. A review of validations of dietary assessment methods. Am. J. Epidemiol. 1982; 115: 492-505.

19 Tucker JA, Vuchinich RE, Harris CH, Gavornik MG, Rudd EJ. Agreement between subject and collateral verbal reports of alcohol consumption in older adults. J. Stud. Alcohol 1991; 52: $148-55$.

20 Flegal KM. Agreement between two dietary methods in reported intake of beer, wine, and liquor. J. Stud. Alcohol 1991; 52: 174-9.

21 Poikolainen K, Kärkkäinen P. Nature of questionnaire options affects estimates of alcohol intake. J. Stud. Alcohol 1985; 46: 219-22.

22 Pietinen P, Hartman AM, Haapa E, Rasanen L, Haapakoski J, Palmgren J, et al. Reproducibility and validity of dietary assessment instruments. I. A self-administered food use questionnaire with a portion size picture booklet. $\mathrm{Am}$. J. Epidemiol. 1988; 128: 655-66.

23 Van Leeuwen FE, de Vet HCW, Hayes RB, van Staveren WA, West CE, Hautvast JG. An assessment of the relative validity of retrospective interviewing for measuring dietary intake. Am. J. Epidemiol. 1983; 118: 752-8.

24 Riboli E, Carpele E. Evaluation of dietary assessment methods. Ital. J. Gastroenterol. 1986; 18: 5-10.

25 Dwyer JT, Gardner J, Halvorsen K, Krall EA, Cohen A, Valadian I. Memory of food intake in the distant past. Am. J. Epidemiol. 1989; 130: 1033-46.

26 Lemmens PH, Knibbe RA, Tan F. Weekly recall and diary dietary estimates of alcohol consumption in a general population survey. J. Stud. Alcohol 1988; 49: 131-5.

27 Feunekes GI, van't Veer P, van Staveren WA, Kok FJ. Alcohol intake assessment: the sober facts. Am. J. Epidemiol. 1999; 150: $105-12$.

28 Williams GD, Aitken SS, Malin H. Reliability of self-reported alcohol consumption in a general population survey. J. Stud. Alcohol 1985; 46: 223-7.

29 Hill A, Roberts J, Ewings P, Gunnell D. Non-response bias in a lifestyle survey. J. Public Health Med. 1997; 19: 203-7.

30 Whitfield JB. Gamma glutamyl transferase. Crit. Rev. Clin. Lab. Sci. 2001; 38: 263-355. 